\title{
INTRAVESICAL RESINIFERATOXIN VERSUS BOTULINUM-A TOXIN INJECTIONS FOR NEUROGENIC DETRUSOR OVERACTIVITY: A PROSPECTIVE RANDOMIZED STUDY
}

\author{
ANTONELLA GIANNANTONI,* SAVINO M. DI STASI, ROBERT L. STEPHEN, VITTORIO BINI, \\ ELISABETTA COSTANTINI AND MASSIMO PORENA
}

From the Department of Urology, University of Perugia, Perugia, Department of Urology, Tor Vergata University, Rome, and Physion Laboratories, Medolla, Italy

\section{ABSTRACT}

Purpose: We investigated the effectiveness and safety of intravesical resiniferatoxin (Sigma Chemical Co., St. Louis, Missouri) and botulinum-A toxin injections into the detrusor muscle in a group of spinal cord injured patients with neurogenic detrusor overactivity unresponsive to conventional anticholinergic therapy.

Materials and Methods: A total of 25 patients were randomly assigned to receive intravesically $0.6 \mu \mathrm{M}$ resiniferatoxin in $50 \mathrm{ml}$ of $0.9 \% \mathrm{NaCl}$ or injections into the detrusor muscle of 300 units botulinum A-toxin diluted in $30 \mathrm{ml} 0.9 \% \mathrm{NaCl}$. Clinical evaluation and urodynamics were performed at baseline, and at 6,12 and 18 months after treatment.

Results: In both arms there was a significant decrease in catheterization and incontinent episodes, and a significant increase in first detrusor contraction and maximum bladder capacity at 6, 12 and 18-month followup. There were no local side effects with either treatment. Botulinum-A toxin induced a significant decrease in the frequency of daily incontinence episodes $(p<0.05)$, a significant increase in first uninhibited detrusor contraction $(p<0.01)$ in maximum bladder capacity $(\mathrm{p}<0.01)$, and a significant decrease in maximum pressure of uninhibited detrusor contractions $(p<0.01)$ compared to resiniferatoxin at 6, 12 and 18-month followup.

Conclusions: In spinal cord injured patients with refractory neurogenic detrusor overactivity, intravesical resiniferatoxin and botulinum-A toxin injections into the detrusor muscle provided beneficial clinical and urodynamic results with decreases in detrusor overactivity and restoration of urinary continence in a large proportion of patients. Botulinum-A toxin injections provided superior clinical and urodynamic benefits compared to those of intravesical resiniferatoxin.

KEY WoRDs: capsaicin, botulinum toxins; bladder, neurogenic

New strategies for treating refractory neurogenic detrusor overactivity include intravesical administration of vanilloid agents and botulinum-A toxin injections into the detrusor muscle. ${ }^{1}$ Resiniferatoxin (RTX) has been shown to inhibit transmission from the bladder along the afferent $\mathrm{C}$ fibers, providing durable, months long beneficial effects in $60 \%$ to $70 \%$ of patients with neurogenic detrusor overactivity. ${ }^{2-5}$ Botulinum-A toxin selectively blocks acetylcholine release from nerve endings and has recently been used for inhibiting parasympathetic neural transmission into the detrusor muscle in patients with neurogenic detrusor overactivity and urinary incontinence with long lasting and eventually reversible beneficial effects. ${ }^{6,7}$ Preliminary results have shown a significant increase in bladder capacity and in urinary continence with both treatments. However, to our knowledge there are no clinical studies comparing intravesical RTX and botulinum-A toxin injections into the detrusor muscle. We investigated the effectiveness and safety of intravesical RTX and of botulinum-A toxin injections, comparing clinical and urodynamic effects over long-term followup in a group of spinal cord injured patients with neurogenic detrusor overactivity unresponsive to conventional anticholinergic therapy.

Accepted for publication January 16, 2004.

Study received ethics committee approval.

* Correspondence: Via Torrice n. 4, 00189 Rome, Italy (telephone: 390755731248; FAX: 390755726123; e-mail: uropg@rdn.it).

\section{MATERIALS AND METHODS}

Patient characteristics. Enrolled in this study were 18 males and 7 females with chronic spinal cord injury. Mean age \pm SD was $38.4 \pm 12.5$ years and mean disease duration was $41.8 \pm 16.5$ months. Neurological clinical evaluation followed American Spinal Injury Association classification, 8 a type A impairment was observed in 16 patients and a type $B$ was detected in 9 . A total of 20 patients had a lesion at the thoracic level while the remaining patients showed a cervical injury. The study was approved by the ethics committee, all patients were informed about the scientific nature of the study and provided written consent.

Preliminary assessment. Standard evaluation included a history, physical examination, serum chemistries, urinalysis, urine culture and imaging assessment of urinary tract by ultrasound, excretory urography and cystourethrography. Baseline urodynamics followed International Continence Society standards $^{9}$ and was performed 1 month before commencing the study. Maximum pressure of uninhibited detrusor contractions (UDC), the lowest volume (threshold) at which contractions occurred and maximum bladder capacity were recorded. Inclusion criteria were unacceptable clinical (patient driven) and urodynamic suppression of detrusor activity (author assessment) by oral and intravesical oxybutynin, intolerable anticholinergic side effects, normal renal function, no vesicoureteral reflux and a normal upper urinary tract. Patients with myasthenia gravis and other diseases affecting cholinergic action, pregnancy and concomi- 
tant use of aminoglycosides were excluded from study. All patients performed clean intermittent catheterization and were taking oral anticholinergics.

Study plan. A total of 25 patients fulfilling all criteria were selected and randomized by commercially available software into 2 groups. Patients in group 1 (13) received intravesical administrations of RTX for 45 minutes through a polyvinyl chloride catheter with real-time cystometrography as outpatients in our urology department. Resiniferatoxin $1 \mathrm{mg}$ was dissolved in $0.9 \%$ sodium chloride as a stock solution, sterile filtered and divided into aliquots which were stored at $-80 \mathrm{C}$. On the day of use instillations were prepared by diluting the stock solutions with $0.9 \%$ sodium chloride to the requisite concentration of $0.6 \mu \mathrm{M}$ in $50 \mathrm{ml}$ of $0.9 \% \mathrm{NaCl}$. Patients in group 2 (12) were injected with 300 units of commercially available botulinum-A toxin diluted in $30 \mathrm{ml} 0.9 \% \mathrm{NaCl}$ into the detrusor muscle (30 sites, sparing the trigone) under cystoscopic control. ${ }^{6}$ In 6 patients (4 with lesions above T5 and prone to autonomic dysreflexia, and 2 with increased spasticity and leg spasms) the procedure was performed with the patient under spinal anesthesia on an inpatient basis. The remaining procedures were performed with the patient under sedation only.

Patients were asked to decrease the daily dosage of anticholinergics within 15 days of the beginning of treatment. RTX and botulinum-A toxin treatments were repeated after confirmation that frequency of daily incontinence episodes, and catheterization and urodynamic parameters had returned to baseline values. Urinary symptoms were assessed with a voiding diary maintained by patients for 30 days before commencing the study and for 2 days per week throughout followup. Patients recorded the number of daily catheterizations and episodes of incontinence. Urodynamic studies were repeated at 6,12 and 18-month followup, and uninhibited detrusor contractions threshold, maximum pressure and maximum cystometric bladder capacity were recorded. Local and/or systemic side effects were noted during and after treatments.

Data analysis. Statistical analysis was performed using the Friedman, Wilcoxon and Mann-Whitney tests for nonparametric data. The Bonferroni correction was applied to post hoc multiple comparisons and the procedure of Cuzick ${ }^{10}$ was used to calculate the $\mathrm{z}$ test for trend. Statistical significance was set at $p<0.05$. All data analyses were performed using SPSS release 10.1.1 for Windows (SPSS Inc., Chicago, Illinois, 1999).

\section{RESULTS}

Pretreatment assessment. Initially 10 patients in group 1 and 12 in group 2 had incontinence episodes, and used pads or external collecting devices. There were no significant differences in patients who received RTX and botulinum-A toxin with respect to the frequency of catheterization, daily incontinent episodes (see table) or the number using pads and/or external devices. Urodynamics revealed detrusor hyperreflexia, with detrusor-sphincter dyssynergia in 17, decreased bladder capacity and urinary incontinence in all patients. There was no significant difference in bladder capacity, uninhibited detrusor contraction threshold or maximum pressure of contractions in patients assigned to the RTX or botulinum-A toxin arms (see table). In group 1, 7 patients and in group 2, 6 patients reported signs and symptoms of autonomic dysreflexia which were also detected during urodynamic evaluation.

Followup. RTX Arm: Mean followup was $14.8 \pm 3$ months, mean number of instillations per patient was $8.6 \pm 1.9$ and mean time between 2 consecutive instillations was $51.6 \pm 8.2$ days. Clinical results are shown in the table. At 6,12 and 18 months mean catheterization and incontinent episodes were significantly decreased compared to baseline $(p<0.05$ and $\mathrm{p}<0.001$, respectively), and 5 patients achieved complete urinary continence which was maintained during followup. On urodynamics (see table) we detected a significant increase in the uninhibited detrusor contractions threshold, and in maximum bladder capacity at 6,12 and 18-month followup ( $p<0.001)$. We did not observe any significant change in maximum pressure of uninhibited detrusor contractions during followup compared to baseline. Episodes of autonomic dysreflexia disappeared in 3 patients during urodynamics and the remainder reported an amelioration of the condition during everyday life. We did not observe any local or systemic side effects during or after intravesical instillations. Ten patients continued to take anticholinergics during the entire observation, but with an average decrease of $56.4 \%$ in dosage (in 3 patients, $100 \%$ ).

Botulinum-A Toxin Arm: Mean followup was $14.2 \pm 3.9$ months, the number of treatments per patient was $2.1 \pm 0.7$ and mean time between 2 consecutive injections was $6.8 \pm$ 1.5 months. Clinical results are shown in the table. At 6 months mean catheterization and incontinent episodes were significantly decreased compared to baseline $(\mathrm{p}<0.001$ and $\mathrm{p}<0.001$, respectively). At 6 and 12 months 9 patients were completely continent and 6 of them followed to 18 months remained continent. In the remaining patients we observed a decrease in daily incontinent episodes. At 6, 12 and 18 months urodynamic evaluation (see table) revealed a significant increase in the uninhibited detrusor contractions threshold $(\mathrm{p}<0.001)$ and in maximum bladder capacity $(p<0.001)$, and a significant decrease in maximum pressure of uninhibited detrusor contractions $(p<0.001)$ as compared

Urodynamics in 13 patients in RTX arm and in 12 patients in botulinum-A toxin arm

\begin{tabular}{|c|c|c|c|c|}
\hline & \multicolumn{4}{|c|}{ Mean \pm SD } \\
\hline & Before Treatment & 6 Mos & 12 Mos & 18 Mos \\
\hline \multicolumn{5}{|c|}{ No. catheterizations daily: } \\
\hline RTX & $5.7 \pm 1.1$ & $4.9 \pm 0.7^{*}$ & $4.7 \pm 0.6^{*}$ & $4.8 \pm 0.6^{*}$ \\
\hline Botulinum-A toxin & $6.0 \pm 0.9$ & $4.6 \pm 0.4 \dagger$ & $4.5 \pm 0.5 \dagger$ & $4.4 \pm 0.5 \dagger$ \\
\hline \multicolumn{5}{|c|}{ No. incontinence episodes daily: } \\
\hline RTX & $5.4 \pm 1.3$ & $2.2 \pm 1.2 \dagger$ & $2.0 \pm 1.3 \dagger$ & $2.0 \pm 1.1 \dagger$ \\
\hline Botulinum-A toxin & $4.8 \pm 1.1$ & $1.4 \pm 1.7 \dagger$ & $0.3 \pm 0.5 \dagger$ & $0.7 \pm 0.9 \dagger$ \\
\hline \multicolumn{5}{|l|}{ UDC threshold (ml): } \\
\hline RTX & $205.5 \pm 69.7$ & $288.7 \pm 83.7 \ddagger$ & $285.5 \pm 85.6 \neq$ & $274.8 \pm 69.3 \ddagger$ \\
\hline Botulinum-A toxin & $190 \pm 48.6$ & $326.3 \pm 80.9 \ddagger, \S$ & $361 \pm 62.7 \ddagger, \S$ & $411.5 \pm 56 \ddagger, \S$ \\
\hline \multicolumn{5}{|c|}{ UDC max pressure $\left(\mathrm{cm} \mathrm{H}_{2} \mathrm{O}\right)$ : } \\
\hline RTX & $70.2 \pm 26$ & $63.1 \pm 26.2$ & $61.4 \pm 24.8$ & $65.1 \pm 22.1$ \\
\hline Botulinum-A toxin & $78.5 \pm 21.5$ & $47 \pm 18.3 \ddagger, \S$ & $45 \pm 10 \ddagger, \S$ & $35.2 \pm 19.5 \ddagger \S$ \\
\hline \multicolumn{5}{|c|}{ Max bladder capacity (ml): } \\
\hline RTX & $223.3 \pm 68.1$ & $329 \pm 72.3 \ddagger$ & $338 \pm 63.7$ 末 & $327.7 \pm 57.8 \ddagger$ \\
\hline Botulinum-A toxin & $211.9 \pm 49.7$ & $370 \pm 79.6 \div, \S$ & $415 \pm 75 \ddagger, \S$ & $450.6 \pm 53.2+, \S$ \\
\hline
\end{tabular}

*Vs pretreatment $\mathrm{p}<0.05$.

$\dagger$ Vs pretreatment $\mathrm{p}<0.001$.

$¥$ Vs pretreatment $\mathrm{p}<0.001$.

$\S$ Vs RTX p $<0.01$. 
to before treatment. Episodes of autonomic dysreflexia disappeared during urodynamics in 4 patients at 6, 12 and 18 months, and patients did not report any episode during everyday life. We did not observe any local side effects during and after intra-detrusor injections. One patient complained of mild asthenia soon after the first treatment, which persisted for 10 days. There were 7 patients who continued to take anticholinergics during followup but with the dosage decreased by an average of $62.5 \%$.

RTX Versus Botulinum-A Toxin: There was a significant decrease in the frequency of daily incontinence episodes in the botulinum-A toxin arm compared to RTX at 6, 12 and 18-month followup $(\mathrm{p}<0.05)$. Furthermore, we detected a significant increase in the uninhibited detrusor contractions threshold $(\mathrm{p}<0.01)$ and in maximum bladder capacity $(p<0.01)$, and a significant decrease in maximum pressure of uninhibited detrusor contractions $(\mathrm{p}<0.01)$ in the botulinum-A toxin arm compared to RTX at 6, 12 and 18month followup.

\section{DISCUSSION}

During the last decade there have been many efforts to provide therapeutic alternatives to standard anticholinergics in the treatment of neurogenic detrusor overactivity. Administration of intravesical resiniferatoxin and botulinum-A toxin injections into the detrusor muscle represent new therapeutic options for patients who do not respond to or who cannot tolerate standard anticholinergic regimens. ${ }^{1}$ They differ largely in chemical structure and mechanisms of action, and techniques for administration are not fully standardized.

The reorganization of the micturition reflex after the interruption of spinal cord pathways induces $\mathrm{C}$ fiber afferents to become mechanoreceptors which initiate reflex voiding. ${ }^{11}$ This process is the rationale for using intravesical resiniferatoxin to decrease the number of $\mathrm{C}$ fiber afferents into the bladder wall, and to suppress detrusor overactivity and urinary incontinence. ${ }^{2}$ Several studies have been done on the treatment of neurogenic ${ }^{3-5}$ and nonneurogenic detrusor overactivity, ${ }^{12}$ and they all indicate that intravesical administration of RTX is safe and effective in increasing the bladder volume at which uninhibited detrusor contractions occur and increasing maximum bladder capacity. Our failure to induce significant change in the maximum pressure of UDC is a cause for concern. Although this effect can be anticipated from the mode of action of RTX (inhibition of the afferent arm of the reflex arc) when associated with detrusor-sphincter dyssynergia renal damage may occur in the long term. Therefore, such patients must be closely observed for evidence of upper urinary tract dilation and remedial measures must be used to reverse or arrest this situation.

More recently botulinum-A toxin injections in the detrusor muscle have been introduced.6,7 Botulinum-A toxin inhibits acetylcholine release at the presynaptic neuromuscular junction resulting in regional decreased muscle contractility and atrophy at the site of injections. ${ }^{13}$ Schurch et al reported preliminary results in spinal cord injured patients with refractory detrusor hyperreflexia and found that botulinum-A toxin injected into the detrusor muscle significantly increases mean reflex volume and maximum bladder capacity without any local or systemic side effects. ${ }^{6}$ The duration of bladder paresis is at least 9 months, after which repeat injections may be required. Under the conditions imposed in this study, botulinum-A toxin injection into the detrusor muscle is superior to intravesical resiniferatoxin in terms of urodynamic results and clinical benefits in patients with neurogenic detrusor overactivity over long-term followup. However, there are several facets to both treatments that must be addressed.

$R T X$. First, intravesical administration provides a concentration gradient for RTX which diffuses into and across the urothelium (Fick's first law). However, in patients with detrusor overactivity urothelium are not all equal. The original disease, duration of disease, level of spinal cord lesions, number and severity of urinary tract infection will all cause variations in urothelial permeability and, thus, in the amount of RTX reaching the target sites. Second, a standard baseline dose (a function of concentration, volume and dwell time) has not been defined and concentrations varying 100fold have been reported as effective. ${ }^{2-5}$ Third, the optimum vehicle, water based or ethanol and water, has not been defined. Eventually there is no reason that standardized vehicles, volumes and dwell times cannot be achieved. However, it is obvious that RTX concentrations must eventually be individualized for each patient. The problems associated with lipophilic RTX are succinctly described by Szallasi and Fowler. ${ }^{14}$

Botulinum-A toxin. Intravesical injections bypass the transurothelial transport uncertainties and all of an injected dose is at or near the target site. This makes the standardization of an effective dose much easier than is the case for RTX and it is probable that a standard routine of injections will suit most patients. An important concern is a possible increase in drug tolerance after repeat injections, a phenomenon which has not been observed in the present study or previously reported by others. ${ }^{15}$

Another issue is that RTX administration is a simple procedure that can be performed almost anywhere, even in patient homes. Botulinum-A toxin injections require some form of anesthesia or sedation for the patient, with special equipment and a skilled endoscopist to ensure accurate placement of injections. Therefore, the choice of one treatment or the other depends on the facilities available and patient choice.

\section{CONCLUSIONS}

In spinal cord injured patients with neurogenic detrusor overactivity refractory to standard anticholinergic treatment, intravesical resiniferatoxin and botulinum-A toxin injections provided beneficial clinical and urodynamic results with a decrease in detrusor overactivity and restoration of urinary continence in a large proportion of patients. Botulinum-A toxin injection into the detrusor muscle provided superior clinical and urodynamic benefits compared to those of intravesical resiniferatoxin.

\section{REFERENCES}

1. Yoshimura, N. and Chancellor, M. B.: Current and future pharmacological treatment for overactive bladder. J Urol, 168: 1897, 2002

2. Chancellor, M. B. and de Groat, W. C.: Intravesical capsaicin and resiniferatoxin therapy: spicing up the ways to treat the overactive bladder. J Urol, 162: 3, 1999

3. Lazzeri, M., Spinelli, M., Beneforti, P., Zanollo, A. and Turini, D.: Intravesical resiniferatoxin for the treatment of detrusor hyperreflexia refractory to capsaicin in patients with chronic spinal cord diseases. Scand J Urol Nephrol, 32: 331, 1998

4. Silva, C., Rio, M. E. and Cruz, F.: Desensitization of bladder sensory fibers by intravesical resiniferatoxin, a capsaicin analog: long-term results for the treatment of detrusor hyperreflexia. Eur Urol, 38: 444, 2000

5. Giannantoni, A., Di Stasi, S. M., Stephen, R. L., Navarra, P., Scivoletto, G., Mearini, E. et al: Intravesical capsaicin versus resiniferatoxin in patients with detrusor hyperreflexia: a prospective randomized study. J Urol, 167: 1710, 2002

6. Schurch, B., Stöhrer, M., Kramer, G., Schmid, D. M., Gaul, G. and Hauri, D.: Botulinum-A toxin for treating detrusor hyperreflexia in spinal cord injured patients: a new alternative to anticholinergic drugs? Preliminary results. J Urol, 164: 692, 2000

7. Schulte-Baukloh, H., Michael, T., Sturzebecher, B. and Knispel, H. H.: Botulinum-a toxin detrusor injection as a novel approach in the treatment of bladder spasticity in children with neurogenic bladder. Eur Urol, 44: 139, 2003 
8. Ditunno, J. F., Jr., Young, W., Donovan, W. H. and Creasey, G.: The international standards booklet for neurological and functional classification of spinal cord injury. American Spinal Injury Association. Paraplegia, 32: 70, 1994

9. Stohrer, M., Goepel, M., Kondo, A., Kramer, G., Madersbacher, H., Millard, R. et al: The standardization of terminology in neurogenic lower urinary tract dysfunction: with suggestions for diagnostic procedures. International Continence Society Standardization Committee. Neurourol Urodyn, 18: 139, 1999

10. Cuzick, J.: A Wilcoxon-type test for trend. Stat Med, 4: 87, 1985

11. Yoshimura, N.: Bladder afferent pathway and spinal cord injury: possible mechanisms inducing hyperreflexia of the urinary bladder. Prog Neurobiol, 57: 583, 1999
12. Silva, C., Ribeiro, M. J. and Cruz, F.: The effect of intravesical resiniferatoxin in patients with idiopathic detrusor instability suggests that involuntary detrusor contractions are triggered by C-fiber input. J Urol, 168: 575, 2002

13. Duchen, L. W.: Changes in motor innervation and cholinesterase localization induced by botulinum toxin in skeletal muscle of the mouse: differences between fast and slow muscles. J Neurol Neurosurg Psychiatry, 33: 40, 1970

14. Szallasi, A. and Fowler, C. J.: After a decade of intravesical vanilloid therapy: still more questions than answers. Lancet Neurol, 1: 167, 2002

15. Leippold, T., Reitz, A. and Schurch, B.: Botulinum toxin as a new therapy option for voiding disorders: current state of the art. Eur Urol, 44: 165, 2003 